CARPATHIAN J. MATH.

Volume 37 (2021), No. 1,

Pages $101-108$

\title{
Some inequalities related to Ky Fan inequality on time scales
}

\author{
CRistian Dinu ${ }^{1}$, DANiela DĂnCIUlescu ${ }^{1}$ and Alexandru ŢUGUi ${ }^{2}$
}

\begin{abstract}
In the framework of the time scales, we present some improved versions of the Ky-Fan inequality for functions, using some weights that take both negative and positive values. In addition, we consider a perspective to use these models in the economic context, since Economics offers many opportunities for time scales applications. Thus, time scales provide not only an unification for discrete and continuous mathematics, but also for the discrete and continuous approaches in economic studies.
\end{abstract}

\section{INTRODUCTION}

The dynamic derivatives on time scales provide a theory that can unify and, also extend the traditional differential and difference equations. It can be considered an unification of the discrete theory with the continuous theory and it was introduced by Stefan Hilger in [18]. A combined dynamic derivative, so called $\nabla_{\alpha}$ (diamond- $\alpha$ ) dynamic derivative, was introduced as a linear combination of $\Delta$ and $\nabla$ dynamic derivatives on time scales. This diamond- $\alpha$ dynamic derivative reduces to the $\Delta$ derivative for $\alpha=1$ and to the $\nabla$ derivative for $\alpha=0$. For what follows, it is assumed that the basic notions of the time scales calculus are known. We can refer the reader to [1, 4, 18, 21, 22].

One of the most important field where we can apply time scale calculus is Economics. Both discrete and continuous economic models can be found in the standard studies. For instance, the consumption of a person can be modeled using the discrete case when the income received by the consumer comes in a period of time and so, he can modify his behavior according to his decisions regarding the consumption and the saving during that period. Thus, we assume that all the decisions are made at some time spaced intervals.

An interesting counterpart of the arithmetic-geometric mean inequality is the inequality of Ky Fan, that can be stated as follows:

Theorem 1.1. If $0<x_{i} \leq \frac{1}{2}$, for $i=1, \ldots, n$, then

$$
\left[\prod_{i=1}^{n} x_{i} / \prod_{i=1}^{n}\left(1-x_{i}\right)\right]^{\frac{1}{n}} \leq \sum_{i=1}^{n} x_{i} / \sum_{i=1}^{n}\left(1-x_{i}\right)
$$

with equality only if $x_{1}=\ldots=x_{n}$.

If $x=\left(x_{1}, \ldots, x_{n}\right)$ and $w=\left(w_{1}, \ldots, w_{n}\right)$ are two $n$-tuples of numbers and $w_{k} \geq 0$ for each $k$ and $\sum_{k=1}^{n} w_{k}=1$, then the arithmetic, geometric and harmonic means of $x$ with the weight $w$ are defined as follows

Received: 14.06.2019. In revised form: 20.01.2021. Accepted: 27.01.2021

2010 Mathematics Subject Classification. 26D15, 39A13.

Key words and phrases. Time scales, convex function, dynamic derivatives, Ky Fan inequality, Jensen inequality etc.

Corresponding author: Daniela Dănciulescu; danadanciulescu@gmail.com 


$$
A_{n}(x, w)=\sum_{k=1}^{n} w_{k} x_{k}, \quad G_{n}(x, w)=\prod_{k=1}^{n} x_{k}^{w_{k}}, \quad H_{n}(x, w)=\frac{1}{\sum_{k=1}^{n} w_{k} / x_{k}} .
$$

The inequality (1.1) works also in the weighted case, as it was proved. Using the above notations, we have:

$$
\frac{G_{n}(x, w)}{G_{n}(1-x, w)} \leq \frac{A_{n}(x, w)}{A_{n}(1-x, w)}
$$

and also the complementary inequality (see [25]),

$$
\frac{H_{n}(x, w)}{H_{n}(1-x, w)} \leq \frac{G_{n}(x, w)}{G_{n}(1-x, w)} .
$$

The above inequalities were improved by Dragomir and Scarmozzino in [14]. S. Simić gave a converse version of Ky Fan inequality in [23].

C. Dinu presented a complete weighted version of the Jensen inequality for weights that are allowed to take some negative values in [12].

Theorem 1.2. [Theorem 2 in [12]]. Let $a, b \in \mathbb{T}$ and $m, M \in \mathbb{R}$. If $g \in C\left([a, b]_{\mathbb{T}},[m, M]\right)$ and $w \in C\left([a, b]_{\mathbb{T}}, \mathbb{R}\right)$ with $\int_{a}^{b} w(t) \diamond_{\alpha} t>0$, then the following assertions are equivalent:

(i) $w$ is an $\alpha$-SP weight for $g$ on $[a, b]_{\mathbb{T}}$;

(ii) for every $f \in C([m, M], \mathbb{R})$ convex function, we have

$$
f\left(\frac{\int_{a}^{b} g(t) w(t) \diamond_{\alpha} t}{\int_{a}^{b} w(t) \diamond_{\alpha} t}\right) \leq \frac{\int_{a}^{b} f(g(t)) w(t) \diamond_{\alpha} t}{\int_{a}^{b} w(t) \diamond_{\alpha} t} .
$$

For the concave functions, the above inequality is reversed. Using this version, we improve the inequalities from [14] and [23].

\section{EXTENSION OF THE KY FAN INEQUALITY}

Throughout this paper, let $\mathbb{T}$ be a time scale and $a, b \in \mathbb{T}$. For a function on time scale, we redefine the weighted means and we extend the definitions given in (1.2). A continuous function $w: \mathbb{T} \rightarrow \mathbb{R}$ is called a $\alpha$-weight on $[a, b]_{\mathbb{T}}$, provided that $\int_{a}^{b} w(t) \diamond_{\alpha} t>$ 0 , where $\alpha \in[0,1]$.

Definition 2.1. Let $x:[a, b]_{\mathbb{T}} \rightarrow \mathbb{R}_{+}$be a continuous positive function and $w$ an $\alpha$-weight on $[a, b]_{\mathbb{T}}$. We define:

- the generalized weighted arithmetic mean of the function $x$ on the time scale interval $[a, b]$ of weight $w$ :

$$
A_{[a, b]}(x, w)=\frac{\int_{a}^{b} w(t) x(t) \diamond_{\alpha} t}{\int_{a}^{b} w(t) \diamond_{\alpha} t} ;
$$

- the generalized weighted geometric mean of the function $x$ on the time scale interval $[a, b]$ of weight $w$ :

$$
G_{[a, b]}(x, w)=\exp \left(\frac{\int_{a}^{b} w(t) \ln (x(t)) \diamond_{\alpha} t}{\int_{a}^{b} w(t) \diamond_{\alpha} t}\right) ;
$$


- the generalized weighted harmonic mean of the function $x$ on the time scale interval $[a, b]$ of weight $w$ :

$$
H_{[a, b]}(x, w)=\frac{\int_{a}^{b} w(t) \diamond_{\alpha} t}{\int_{a}^{b} w(t) / x(t) \diamond_{\alpha} t} .
$$

Example 2.1. (i) If $\mathbb{T}=\mathbb{R}$ then, for the $\alpha$-weight $w: \mathbb{R} \rightarrow \mathbb{R}$, (that is $\int_{a}^{b} w(t) \mathrm{d} t>0$ ) we have

$$
\begin{gathered}
A_{[a, b]}(x, w)=\frac{\int_{a}^{b} w(t) x(t) \mathrm{d} t}{\int_{a}^{b} w(t) \mathrm{d} t} ; \\
G_{[a, b]}(x, w)=\exp \left(\frac{\int_{a}^{b} w(t) \ln (x(t)) \mathrm{d} t}{\int_{a}^{b} w(t) \mathrm{d} t}\right) ; \\
H_{[a, b]}(x, w)=\frac{\int_{a}^{b} w(t) \mathrm{d} t}{\int_{a}^{b} w(t) / x(t) \mathrm{d} t} .
\end{gathered}
$$

(ii) If $\mathbb{T}=\mathbb{Z}, a=1, b=n+1$ and $\alpha=1$, we define $w(i)=w_{i}$ and $x(i)=x_{i}$. The condition of 1-weight for $w$ means that $\sum_{i=1}^{n} w_{i}>0$. Then, we have

$$
\begin{gathered}
A_{[a, b]}(x, w)=A_{n}(x, w)=\frac{\sum_{i=1}^{n} w_{i} x_{i}}{\sum_{i=1}^{n} w_{i}} ; \\
G_{[a, b]}(x, w)=G_{n}(x, w)=\sum_{i=1}^{n} \sqrt[w_{i}]{\prod_{i=1}^{n} x_{i}^{w_{i}}} ; \\
H_{[a, b]}(x, w)=H_{n}(x, w)=\frac{\sum_{i=1}^{n} w_{i}}{\sum_{i=1}^{n} w_{i} / x_{i}} .
\end{gathered}
$$

Remark 2.1. The generalized $A M-G M-H M$ inequality is also true for the generalized weighted means. We have,

$$
H_{[a, b]}(x, w) \leq G_{[a, b]}(x, w) \leq A_{[a, b]}(x, w),
$$

while for the proof of the right hand of this inequality, we use Theorem 1.2, taking the concave function $f(t)=\ln (t)$ and $g=x$. For the left side, we use the same function $f$ and $g=1 / x$.

Our main results improve this inequality.

Theorem 2.3. Let $x:[a, b]_{\mathbb{T}} \rightarrow[m, M]$ be a continuous positive function such that $0<m \leq$ $x(t) \leq M \leq \frac{\gamma}{2}, \gamma>0$ and $w$ be an $\alpha$-weight on $[a, b]_{\mathbb{T}}$. Then

$$
\begin{aligned}
\frac{A_{[a, b]}(x, w)}{G_{[a, b]}(x, w)} & \geq\left(\frac{A_{[a, b]}(x, w)}{G_{[a, b]}(x, w)}\right)^{M^{2} /(\gamma-M)^{2}} \geq \frac{A_{[a, b]}(\gamma-x, w)}{G_{[a, b]}(\gamma-x, w)} \\
& \geq\left(\frac{A_{[a, b]}(x, w)}{G_{[a, b]}(x, w)}\right)^{m^{2} /(\gamma-m)^{2}} \geq 1 .
\end{aligned}
$$

Particulary,

$$
\frac{A_{[a, b]}(x, w)}{A_{[a, b]}(\gamma-x, w)} \geq \frac{G_{[a, b]}(x, w)}{G_{[a, b]}(\gamma-x, w)} .
$$


Proof. Using an idea of Dragomir and Scarmozzino from [14] in the framework of $\alpha$ positive weights, we get some interesting inequalities for not necessarily positive weights.

The remark that $\frac{A_{[a, b]}(x, w)}{G_{[a, b]}(x, w)} \geq 1$, and the fact that $m, M \in\left(0, \frac{\gamma}{2}\right]$, lead us to the first and the last inequality in (2.10). is

We define the function $f:(0, \gamma) \rightarrow \mathbb{R}$ by $f(t)=\ln \frac{\gamma-t}{t}+c \ln t$, whose second derivative

$$
f^{\prime \prime}=\frac{\gamma(\gamma-2 t)}{[t(\gamma-t)]^{2}}-\frac{c}{t^{2}}=\frac{1}{t^{2}}\left[\frac{\gamma(\gamma-2 t)}{(\gamma-t)^{2}}-c\right], \quad t \in(0, \gamma)
$$

is positive on its domain.

Using the function $g:(0, \gamma) \rightarrow \mathbb{R}, g(t)=\frac{\gamma(\gamma-2 t)}{(\gamma-t)^{2}}$, which is monotonically strictly decreasing on $(0, \gamma)$ we get, for every $m \leq t \leq M$,

$$
\frac{\gamma(\gamma-2 M)}{(\gamma-M)^{2}}=g(M) \leq g(t) \leq g(m)=\frac{\gamma(\gamma-2 m)}{(\gamma-m)^{2}}
$$

that is, the function $f$ is strictly convex on $(m, M)$ for $c \leq \frac{\gamma(\gamma-2 M)}{(\gamma-M)^{2}}$.

Applying the generalized Jensen theorem 1.2 for the function $f:(m, M) \rightarrow \mathbb{R}, f(t)=$ $\ln \frac{\gamma-t}{t}+c \ln t$, with $c \leq \frac{\gamma(\gamma-2 M)}{(\gamma-M)^{2}}$, we get

$$
\begin{aligned}
& \ln \left(\frac{\gamma-\frac{\int_{a}^{b} w(t) x(t) \diamond_{\alpha} t}{\int_{a}^{b} w(t) \diamond_{\alpha} t}}{\frac{\int_{a}^{b} w(t) x(t) \diamond_{\alpha} t}{\int_{a}^{b} w(t) \diamond_{\alpha} t}}\right)+c \ln \left(\frac{\int_{a}^{b} w(t) x(t) \diamond_{\alpha} t}{\int_{a}^{b} w(t) \diamond_{\alpha} t}\right) \\
& \quad=f\left(\frac{\int_{a}^{b} w(t) x(t) \diamond_{\alpha} t}{\int_{a}^{b} w(t) \diamond_{\alpha} t}\right) \leq \frac{\int_{a}^{b} w(t) f(x(t)) \diamond_{\alpha} t}{\int_{a}^{b} w(t) \diamond_{\alpha} t} \\
& =\frac{1}{\int_{a}^{b} w(t) \diamond_{\alpha} t}\left(\int_{a}^{b} w(t) \ln \left(\frac{\gamma-x(t)}{x(t)}\right) \diamond_{\alpha} t+c \int_{a}^{b} w(t) \ln (x(t)) \diamond_{\alpha} t\right) .
\end{aligned}
$$

That can be stated as

$$
\ln \left(\frac{A_{[a, b]}(\gamma-x, w)}{A_{[a, b]}(x, w)}\right)+c \ln \left(A_{[a, b]}(x, w)\right) \leq \ln \left(\frac{G_{[a, b]}(\gamma-x, w)}{G_{[a, b]}(x, w)}\right)+c \ln \left(G_{[a, b]}(x, w)\right),
$$

which is equivalent to

$$
\ln \left(\frac{G_{[a, b]}(x, w)}{A_{[a, b]}(x, w)}\right)^{c} \geq \ln \left(\frac{A_{[a, b]}(\gamma-x, w)}{A_{[a, b]}(x, w)} / \frac{G_{[a, b]}(\gamma-x, w)}{G_{[a, b]}(x, w)}\right)
$$

or,

$$
\left(\frac{G_{[a, b]}(x, w)}{A_{[a, b]}(x, w)}\right)^{c-1} \geq \frac{A_{[a, b]}(\gamma-x, w)}{G_{[a, b]}(\gamma-x, w)} .
$$

Replacing $c$ in (2.12) by its maximal value, that is $c=\frac{\gamma(\gamma-2 M)}{(\gamma-M)^{2}}$, we obtain

$$
\left(\frac{G_{[a, b]}(x, w)}{A_{[a, b]}(x, w)}\right)^{\frac{\gamma(\gamma-2 M)}{(\gamma-M)^{2}}-1} \geq \frac{A_{[a, b]}(\gamma-x, w)}{G_{[a, b]}(\gamma-x, w)}
$$

and this yields the second inequality in (2.10).

In the same manner, we define the function $h(t)=d \ln t-\ln \left(\frac{\gamma-t}{t}\right)$, which is convex on $(m, M)$ if $d \geq \frac{\gamma(\gamma-2 m)}{(1-m)^{2}}$ and the proof follows the same steps. 
Our inequality (2.10) is a generalization of the main results from [14] and [15].

Remark 2.2. (i) If $\mathbb{T}=\mathbb{Z}, a=1, b=n+1, \alpha=1$, defining $w(i)=w_{i}$ and $x(i)=x_{i}$ with $\sum_{i=1}^{n} w_{i}>0$ and $x_{i} \in[m, M] \subset(0, \gamma / 2]$ for all $i \in\{1, \ldots, n\}$, then, we have

$$
\begin{aligned}
\frac{A_{n}(x, w)}{G_{n}(x, w)} & \geq\left(\frac{A_{n}(x, w)}{G_{n}(x, w)}\right)^{M^{2} /(\gamma-M)^{2}} \geq \frac{A_{n}(\gamma-x, w)}{G_{n}(\gamma-x, w)} \\
& \geq\left(\frac{A_{n}(x, w)}{G_{n}(x, w)}\right)^{m^{2} /(\gamma-m)^{2}} \geq 1 .
\end{aligned}
$$

(ii) If $\mathbb{T}=\mathbb{R}$ then, for any $a, b \in \mathbb{R}$, any $\alpha$-weight $w: \mathbb{R} \rightarrow \mathbb{R}$, and for any continuous function $x: \mathbb{R} \rightarrow \mathbb{R}$, with $x([a, b]) \subset[m, M] \subset(0, \gamma / 2]$ we have

$$
\begin{aligned}
\frac{A_{[a, b]}(x, w)}{G_{[a, b]}(x, w)} & \geq\left(\frac{A_{[a, b]}(x, w)}{G_{[a, b]}(x, w)}\right)^{M^{2} /(\gamma-M)^{2}} \geq \frac{A_{[a, b]}(\gamma-x, w)}{G_{[a, b]}(\gamma-x, w)} \\
& \geq\left(\frac{A_{[a, b]}(x, w)}{G_{[a, b]}(x, w)}\right)^{m^{2} /(\gamma-m)^{2}} \geq 1 .
\end{aligned}
$$

The inequalities from (2.14) are continuous versions of (2.13) and also they improved continuous versions of Theorem 2 from [15].

Another important result is the following.

Theorem 2.4. Let $x:[a, b]_{\mathbb{T}} \rightarrow[m, M]$ be a continuous positive function such that $0<m \leq$ $x(t) \leq M \leq \frac{\gamma}{2}, \gamma>0$ and $w$ be an $\alpha$-weight on $[a, b]_{\mathbb{T}}$. Then

$$
\frac{1}{H_{[a, b]}(\gamma+x, w)}-\frac{1}{H_{[a, b]}(x, w)} \leq \frac{1}{A_{[a, b]}(\gamma+x, w)}-\frac{1}{A_{[a, b]}(x, w)} .
$$

Proof. Considering $f:(0, \gamma) \rightarrow \mathbb{R}$, a function defined by $f(t)=1 / t-1 /(\gamma+t)$, we have

$$
f^{\prime}=-\frac{1}{t^{2}}+\frac{1}{(\gamma+t)^{2}}, \quad t \in(0, \gamma)
$$

and

$$
f^{\prime \prime}=\frac{2}{t^{3}}-\frac{2}{(\gamma+t)^{3}}
$$

That shows that $f$ is a strictly convex function on $(0, \gamma)$ and we can apply Theorem 1.2 to obtain the inequality from (2.15).

\section{Applications to eCONOMic MODEls. SOME PERSPECTIVES}

Some dynamic optimization problems have a set up in the form that follows: suppose that a customer wants to maximize his lifetime utility subject to some constraints. After each period of life that is considered, he has to make a decision that concerns how much he is about to consume and, at the same time, how much to spend. Therefore, if the consumer would consume more in one day, then in another day he must restrain himself. Precisely, the issue regards not only the consumption, but also the utility that is derived from the consumption. Many economic models are dynamic, thus the results of time scale calculus can apply to economics as well. There are standard economic models that have a continuous or discrete time set-up. These models can be of use for the consumer with his consumption issues. 
We consider the utility function that arrives from consumption. It becomes an optimal control problem, while the solution is a function that models an optimal behavior and it reveals the consumptions for each period for achieving the optimality. Thus, utility can be considered as a value function that the consumer wants to maximize. This function depends on many variables, but its typical dependent variable is consumption, denoted generically by $C$. That is, utility $u(C)$ has the properties $u^{\prime}(C)>0$ and $u^{\prime \prime}(C)<0$ because the consumers always want to increase their consumption, since any additional unit brings positive utility, while an additional unit that is consumed brings less utility than the previous unit consumed within the same period (see [3]). This property is called the Law of Diminishing Marginal Utility.

Following some similar reasoning as in [3], we consider the problem of maximizing the lifetime utility on a time scale, which is the sum of instantaneous utilities

$$
U=\int_{0}^{\sigma(T)} u(C(\rho(s))) \hat{e}_{-\delta}(\rho(s), 0) \diamond_{\alpha} s,
$$

where, the $\diamond_{\alpha}$-exponential function is defined, as in [3], by

$$
\hat{e}_{p}(t, s):=\exp \left(\int_{s}^{t} \hat{\xi}_{\nu(\tau)}(p(\tau)) \diamond_{\alpha} \tau\right)
$$

for every $s, t \in \mathbb{T}$ and the cylinder transformation $\hat{\xi}_{\nu}$ from [5].

By denoting $w(s):=\hat{e}_{-\delta}(\rho(s), 0)$ and $x(s):=u(C(\rho(s)))$ and using the notations in Definition 2.1, we get

$$
U=A_{[0, \sigma(T)]}(x, w) \cdot \int_{0}^{\sigma(T)} \hat{e}_{-\delta}(\rho(s), 0) \diamond_{\alpha} s .
$$

With the above notions, the generalized $A M-G M-H M$ inequality (Remark 2.1), gives an estimation for the lower bound of the utility function, that is

$$
\begin{aligned}
U & \geq G_{[0, \sigma(T)]}(x, w) \cdot \int_{0}^{\sigma(T)} \hat{e}_{-\delta}(\rho(s), 0) \diamond_{\alpha} s \\
& =\exp \left(\frac{\int_{0}^{\sigma(T)} \hat{e}_{-\delta}(\rho(s), 0) \ln (u(C(\rho(s)))) \diamond_{\alpha} s}{\int_{0}^{\sigma(T)} \hat{e}_{-\delta}(\rho(s), 0) \diamond_{\alpha} s}\right) \cdot \int_{0}^{\sigma(T)} \hat{e}_{-\delta}(\rho(s), 0) \diamond_{\alpha} s .
\end{aligned}
$$

A better estimation of this inequality is obtained using our main result Theorem 2.3, since

$$
\frac{A_{[0, \sigma(T)]}(x, w)}{G_{[0, \sigma(T)]}(x, w)}=\frac{\frac{U}{\int_{0}^{\sigma(T)} \hat{e}_{-\delta}(\rho(s), 0) \diamond_{\alpha} s}}{\exp \left(\frac{\int_{0}^{\sigma(T)} \hat{e}_{-\delta}(\rho(s), 0) \ln (u(C(\rho(s)))) \diamond_{\alpha} s}{\int_{0}^{\sigma(T)} \hat{e}_{-\delta}(\rho(s), 0) \diamond_{\alpha} s}\right)}
$$

Notice that the $\nabla_{\alpha}$-exponential function can be seen as a generalized weighted geometric mean of the function $x(\tau)=(1+\mu(\tau) p(\tau))^{\frac{1}{\mu(\tau)}}$. on the time scale interval $[s, t]_{\mathbb{T}}$ of weight $w(\tau)=1$ and one get some upper and lower estimates of the utility function.

For future discussions and generalizations, one might consider useful to consider the utility function $U$ as the sum of the utility functions in all periods, that is

$$
U=\sum_{j=1}^{n} \int_{\sigma\left(T_{j-1}\right)}^{\sigma\left(T_{j}\right)} u_{j}(C(\rho(s))) \hat{e}_{-\delta_{j}}(\rho(s), 0) \diamond_{\alpha} s, \text { where } \mathrm{T}_{0}=0
$$


that leads to the following form in the discrete case

$$
U=\sum_{j=1}^{n} \sum_{s=0}^{T_{j}}\left(\frac{1}{1+\delta_{j}}\right)^{s} u_{j}\left(C_{s}\right),
$$

while, for the continuous case, one gets

$$
U=\sum_{j=1}^{n} \int_{0}^{T_{j}} u_{j}\left(C_{s}\right) e^{-\delta_{j} s} \mathrm{~d} s .
$$

One of the most important economic models is the Economic order quantity model (EOQ), with its generalized form, economic production quantity (EPQ). Various methods have been proposed in order to solve these models, such as: Grubbstrom (1996), (see [17]), Grubbstorm and Erdem (1999), (see [16]), Cardenas-Barron (2001, 2007), (see [6, 7]), Chang et al. (2005), (see [9]). More recently, for these models and other similar ones, Teng et al. (2009), (see [24]), Cardenas-Barron et al. (2011), (see [8]), Lin et al.(2017), (see [19]) offered methods to compute economic order quantities, solved the vendor-buyer integrated inventory system and offered easier ways to obtain the EOQ and EPQ inventory models with backorders.

Related to these models, we can build some general models of type EOQ and EPQ in the economic field of time scales. Using the above results (given by Theorem 2.3) inferior bounds can be given for the appropriate efficient functions. In some hypothesis, by using the corresponding generalized Euler-Lagrange equations, we have that these margins are optimal. All these results are the object of a future research.

Acknowledgments. This work was supported by the grant POCU380/6/13/123990, cofinanced by the European Social Fund within the Sectorial Operational Program Human Capital 2014-2020.

\section{REFERENCES}

[1] Agarwal R. P. and Bohner, M., Basic calculus on time scales and some of its applications, Results Math., 35 (1999), 3-22

[2] Amini, M. R. S. Ferreira, R. A. C. and Torres, D. F. M., Diamond- $\alpha$ Jensen's Inequality on Time Scales and Applications, Journal of Inequalities and Applications, 2008, Article ID 576876, 13 pp., 2008

[3] Ferhan, M., Atici, Daniel C. and Biles, Alex Lebedinsky, An application of time scales to economics, Math Comput Model., 43 (2006), 718-726

[4] Bohner, M. and Peterson, A., Dynamic Equations on Time Scales, An introduction with Applications, Birkhäuser, Boston, 2001

[5] Bohner M. and Peterson A., Advances in Dynamic Equations on Time Scales, Birkhäuser, Boston, 2003

[6] Cardenas-Barron, L. E., The economic production quantity (EPQ) with shortage derived algebraically, International Journal of Production Economics, 70 2001, 289-292

[7] Cardenas-Barron, L. E., Optimizing inventory decisions in a multi-stage multi-customer supply chain: A note, Transportation Research Part E: Logistics and Transportation 43 (2007), No. 5 647-654

[8] Cardenas-Barron, L. E., Wee, H. M. and Blos, M. F., 2011, Solving the vendor-buyer integrated inventory system with arithmetic-geometric inequality, Elsevier, Math Comput Model., 53 (2011), 991-997

[9] Chang, S. K. J., Chuang, J. P. C. and Chen, H. J., 2005 Short comments on technical note - The EOQ and EPQ models with shortages derived without derivatives, Int. J. Prod. Econ., 97 (2005), 241-243

[10] Czinder, P. and Páles, Z., An extension of the Hermite-Hadamard inequality and an application for Gini and Stolarsky means, J. Inequal. Pure and Appl. Math, 5 (2004), No. 2, Article 42, 8 pp.

[11] Dinu, C., Hermite-Hadamard inequality on time scales, Journal of Inequalities and Applications, 2008, Article ID 287947, $24 \mathrm{pp}$.

[12] Dinu, C., A weighted Hermite-Hadamard inequality for Steffensen-Popoviciu and Hermite-Hadamard weights on time scales, Analele Ştiinţifice ale Universităţii "Ovidius", Constanţa, Ser. Mat., 17 (2009), No. 1 77-90

[13] Dragomir, S. S. and Mcandrew, A., Refinements of the Hermite-Hadamard inequality for convex functions, J. Inequal. Pure and Appl. Math, 6 (2005), No. 5, Art. 140. 
[14] Dragomir, S. S. and Scarmozzino, F. P., On the Ky Fan inequality, J. Math. Anal. Appl., 269 (2002), 129-136

[15] Florea, A. and Niculescu, C. P., A Note on the Ky Fan Inequality, An. Univ. Craiova Ser. Mat. Inform., 29 (2002), 47-51

[16] Grubbstrom, R. W. and Erdem, A., The EOQ with backlogging derived without derivatives, Int. J. Prod. Econ., 59 (1999), 529-530

[17] Grubbstrom, R. W., Material requirements planning and manufacturing resource planning. In Warner, M. (Ed.), International Encyclopedia of Business and Management, 4 (1996), Routledge, London, 3400-3420

[18] Hilger, S. Analysis on measure chains - a unified approach to continuous and discrete calculus, Results Math., 35 (1990), 18-56

[19] Lin, S. C., Tuan, H. W. and Peterson, J., Note on "An easy Method to Derive EOQ and EPQ Inventory Models with Backorders", Advances in Analysis, 2 (2017), No. 1, January

[20] Niculescu, C. P. and Persson, L.-E., Convex functions and their applications. A contemporary aproach, SpringerVerlang, Berlin, 2005

[21] Rogers, Jr. J. W. and Sheng, Q., Notes on the diamond- $\alpha$ dynamic derivative on time scales, J. Math. Anal. Appl., 326 (2007), No. 1, 228-241

[22] Sheng, Q, Fadag, M., Henderson, M. and Davis, J. M., An exploration of combined dynamic derivatives on time scales and their applications, Nonlinear Anal. Real World Appl., 7 (2006), No. 3, 395-413

[23] Simić, S., On a converse of Ky Fan inequality, Kragujevac J. Math., 33 (2010), 95-99

[24] Teng, J.T., A simple method to compute economic order quantities. Elsevier, European Journal of Operational Research, 198 (2009), 351-353

[25] Wang, W.-L. and Wang, P.-F., A class of inequalities for symmetric functions, Arta Math. Sinica, 27 (1984), 485-497

\author{
${ }^{1}$ Department of COMPuter SCIENCE \\ UNIVERSITY OF CRAIOVA \\ RESEARCH CENTER FOR ARTificial INTELligenCE “NiCOLAE ŢăNDĂREANU” \\ Str. A. I. CuZA, NO 13, CRAIOVA RO-200585, ROMANIA \\ Email address: danadanciulescu@gmail.com
}

2 A. I. CUZA UniVERSity OF IAŞI

Str. CAROL I, No. 11, IAşi RO-700506, Romania 